\title{
Transcriptomic and proteomic dynamics in the metabolism of a diazotrophic cyanobacterium, Cyanothece sp. PCC 7822 during a diurnal light-dark cycle
}

David Welkie ${ }^{1}$, Xiaohui Zhang ${ }^{1}$, Meng Lye Markillie², Ronald Taylor ${ }^{2}$, Galya Orr ${ }^{2}$, Jon Jacobs ${ }^{3}$, Ketaki Bhide ${ }^{4}$, Jyothi Thimmapuram ${ }^{4}$, Marina Gritsenko ${ }^{3}$, Hugh Mitchell ${ }^{2}$, Richard D Smith ${ }^{3}$ and Louis A Sherman ${ }^{*}$

\begin{abstract}
Background: Cyanothece sp. PCC 7822 is an excellent cyanobacterial model organism with great potential to be applied as a biocatalyst for the production of high value compounds. Like other unicellular diazotrophic cyanobacterial species, it has a tightly regulated metabolism synchronized to the light-dark cycle. Utilizing transcriptomic and proteomic methods, we quantified the relationships between transcription and translation underlying central and secondary metabolism in response to nitrogen free, 12 hour light and 12 hour dark conditions.

Results: By combining mass-spectrometry based proteomics and RNA-sequencing transcriptomics, we quantitatively measured a total of 6766 mRNAs and 1322 proteins at four time points across a 24 hour light-dark cycle. Photosynthesis, nitrogen fixation, and carbon storage relevant genes were expressed during the preceding light or dark period, concurrent with measured nitrogenase activity in the late light period. We describe many instances of disparity in peak mRNA and protein abundances, and strong correlation of light dependent expression of both antisense and CRISPR-related gene expression. The proteins for nitrogenase and the pentose phosphate pathway were highest in the dark, whereas those for glycolysis and the TCA cycle were more prominent in the light. Interestingly, one copy of the psbA gene encoding the photosystem II (PSII) reaction center protein D1 (psbA4) was highly upregulated only in the dark. This protein likely cannot catalyze $\mathrm{O}_{2}$ evolution and so may be used by the cell to keep PSII intact during $\mathrm{N}_{2}$ fixation. The CRISPR elements were found exclusively at the ends of the large plasmid and we speculate that their presence is crucial to the maintenance of this plasmid.

Conclusions: This investigation of parallel transcriptional and translational activity within Cyanothece sp. PCC 7822 provided quantitative information on expression levels of metabolic pathways relevant to engineering efforts. The identification of expression patterns for both mRNA and protein affords a basis for improving biofuel production in this strain and for further genetic manipulations. Expression analysis of the genes encoded on the 6 plasmids provided insight into the possible acquisition and maintenance of some of these extra-chromosomal elements.
\end{abstract}

Keywords: Cyanothece, Cyanobacteria, RNA-Seq, $\mathrm{N}_{2}$ fixation, Proteomics, Butanol, CRISPR

\footnotetext{
* Correspondence: sherman@purdue.edu

'Department of Biological Sciences, Purdue University, West Lafayette, IN, USA

Full list of author information is available at the end of the article
} 


\section{Background}

The interest in utilizing cyanobacterial systems for producing biofuels is increasing as our understanding of metabolism in these photosynthetic microorganisms improves. The ability to utilize carbon acquired from the atmosphere as feedstock along with energy provided by light to produce valuable chemicals and fuels (e.g. ethanol, butanol, hydrogen gas, and fatty acid methyl esters) is even more appealing in diazotrophic strains, since they can grow in the absence of combined nitrogen. Members of the unicellular genus Cyanothece have strong diurnal and circadian cycling of central metabolic processes and the development of a stable genetic manipulation system has been successful in the strain Cyanothece sp. PCC 7822 (herein Cyanothece 7822) [1,2]. In general, members of Cyanothece have well-defined rhythms when grown under $\mathrm{N}_{2}$-fixing conditions, using light/dark or continuous light illumination [3]. During the light phase of growth, light energy is utilized in photosynthesis to secure and store $\mathrm{CO}_{2}$ in intracellular carbohydrate granules. During the dark period, photosynthesis shuts down and respiration utilizes the glucose stored in carbohydrate granules to produce energy and to consume intracellular oxygen. This enables the $\mathrm{O}_{2}$-sensitive nitrogenase to function properly during the dark period. The nitrogenase enzymes allow the cell to fix atmospheric nitrogen, a process which also releases high levels of hydrogen gas [4].

Cyanothece sp. ATCC 51142 (herein Cyanothece 51142) has been studied in great detail and was the first of these unicellular diazotrophs to be sequenced. The high coordination and diurnal oscillations of main metabolic processes such as respiration, photosynthesis, and nitrogen fixation allowed for keen insights into the robust metabolisms of this strain. These characteristics led to a series of transcriptional studies [5-8] and improvements in hydrogen production $[4,9]$. With the advancement of technology, proteomic studies were performed which unveiled a network of interacting protein systems that play off of each other, one system setting up the cellular environment for the next [10-15]. The work described in Aryal et al. [10] was the first to involve proteomics in Cyanothece 51142, in addition to transcriptomics, and demonstrated that many fewer proteins cycled than did transcripts. That study, and a proteomics comparison between Cyanothece 51142 and Cyanothece 7822 [14], indicated that both strains have a higher level of the nitrogenase, respiration and glycogen degradation enzymes in the dark than in the light. Thus, protein cycling throughout the day and night is of functional significance.

Research into Cyanothece 51142 continues, but mutant generation is problematic due to high levels of illegitimate recombination that prevents stable homologous recombination efforts. Due to this, the utility of Cyanothece 51142 as a biological chassis is limited. Min and
Sherman [1] overcame this hurdle in Cyanothece 7822 with the first demonstration of stable genetic modification. This study showcased the difficulty of obtaining stable mutants in most Cyanothece strains and how to improve transformation efficiency by introducing linear, singlestranded, oligonucleotides carrying the desired mutations flanked by fragments homologous to a chromosomal insertion site. More recently, Zhang et al. [2] provided another example of stable genetic modification in Cyanothece 7822 with the generation of a hupL mutant. This mutant led to the unexpected finding that inactivating the uptake hydrogenase in Cyanothece 7822 lowered $\mathrm{H}_{2}$ evolution and nitrogenase activity, thus demonstrating the importance of HupLS in protecting nitrogenase from $\mathrm{O}_{2}$ toxicity.

Transcriptional and proteomic studies on Cyanothece 7822 thus far have compared the metabolic capabilities of Cyanothece 7822 with the other Cyanothece genus members. Recent work by Aryal et al. [14] reported the protein and transcript abundances in Cyanothece 51142 and 7822 under 8 conditions, including nitrogen depletion and mixotrophy. Another study has compared the proteomic expression of five Cyanothece strains in order to determine the metabolic capabilities within the Cyanothece strains [13]. These initial studies have shown that, although the two Cyanothece strains contain quite similar proteomes, they exhibit different protein abundances. These differences suggest the presence of varied cellular strategies in response to altered environmental conditions.

In this study, we utilized transcriptomic and proteomic methods to quantify the relationship between transcription and translation underlying central and secondary metabolism in response to nitrogen fixation at four time points across a $24 \mathrm{~h}$ period consisting of 12 hour light and 12 hour dark. We identified dynamic relationships between transcription and translation, suggesting that there are many levels of regulation in addition to that at the transcriptional level. Our results provide a better understanding of metabolism of Cyanothece 7822 in light and dark conditions and how the metabolic partitioning can be of importance for biofuel production.

\section{Results}

\section{RNA-Sequencing and microarray correlation}

Cyanothece 7822 was grown in nitrogen-free BG-11 media [16] with reduced phosphate [17] under $12 \mathrm{~h}$ light $-12 \mathrm{~h}$ dark conditions for three consecutive days. On the third day of exponential growth, cells were collected for RNAsequencing (RNA-seq) and proteomics at four time points across the light/dark cycle. Material was collected at the beginning of the light period (L0/D12), three hours into the light period (L3), at the end of the light period (L12/ D0), and three hours into the dark period (D3). These time points represent sunrise (L0), mid-morning (L3), sunset (D0) and mid-night (D3) and display the full range 
of expression differences as previously demonstrated in Cyanothece $51142[5,7,8]$.

Since this represented the first RNA-seq analysis for Cyanothece 7822 mRNA transcripts, we compared the results with those from a microarray platform that was constructed as described previously for Cyanothece 51142 and could provide a frame of reference for the results $[7,8]$. Transcriptional expression data was consistent between the two methods. Overall, 6766 and 6539 gene products were detected in total from the RNA-seq and microarray, respectively, and there were $\sim 2$-fold more differentially expressed (DE) genes detected in the RNA-seq at all time points measured (Figure 1 and Additional file 1: Table S1 and Additional file 2: Table S2). This difference was as expected, as similar comparisons have shown that RNA-seq has a lower background signal and a larger dynamic range in expression levels compared to microarrays [18-20].

Statistical comparison indicated that results obtained from the two platforms were highly comparable. Scatter plots were generated using $\log _{2}$ fold change (FC) values of significantly differentially expressed genes (adj-p $\leq 0.05)$ from D0 vs. L0, D3 vs. L0 and L3 vs. L0 (Additional file 3: Figure S1) and the Spearman correlation coefficients for each of the comparisons used to generate the scatter plots were calculated using 'cor' function in R. These coefficient values are listed in Additional file 1: Table S1. Spearman values, especially for significant genes, are comparable with similar studies [18]. With this as a basis, our analysis focused on the RNA-seq data as it provided a more complete analysis of the differential transcription. Additional file 4: Figure S2 is a comparison heat map showing the expression patterns of genes from the main functional categories.

\section{Light-dependent expression of functionally relevant pathways}

The unicellular diazotrophs of the genus Cyanothece follow strong light-dark oscillations for the major physiological functions of nitrogen fixation, respiration and photosynthesis. Thus, we chose sample times that would enable us to determine the type of transcriptional oscillations in Cyanothece 7822 and allow us to compare our results to that of Cyanothece 51142. This permitted us to discuss differential transcription between early (L3 vs. L0 and D3 vs. D0, and late (D0 [L12] vs. L3, and L0 [D12] vs. D3) light and dark periods, respectively.

In Figure 2, the number of genes significantly (adj-p < 0.05 ) upregulated 2-fold ( $>2$ FC) and downregulated 2fold $(<0.5 \mathrm{FC})$ are presented. Prior to the transition into the light and dark periods (D0 and L0, respectively), we see the highest number of differentially expressed genes consistent with the understanding that the cell needs to strongly adjust its enzymatic repertoire to meet the different needs of photosynthesis during the light period, and glycolytic processes and nitrogen fixation during the dark. Observing the expression of core metabolic genes peaking at the onset (e.g., L1 and D1 for Cyanothece 51142) or in the case of Cyanothece 7822 in this study, LO and D0, is a notable characteristic of diurnal gene regulation in cyanobacteria [7]. At D0 (L12) vs. L3, we see the expression of genes for dark metabolic activities such as hydrogenase, nitrogenase, and TCA cycle. At L0 (D12) vs. D3, genes for light metabolism, such as photosynthesis and carbohydrate storage, begin to be upregulated as the cell enters the light phase. Details of each comparison population are provided in Additional file 5: Table S3. This table has a worksheet for each of the comparisons shown in the Venn diagram, identifying

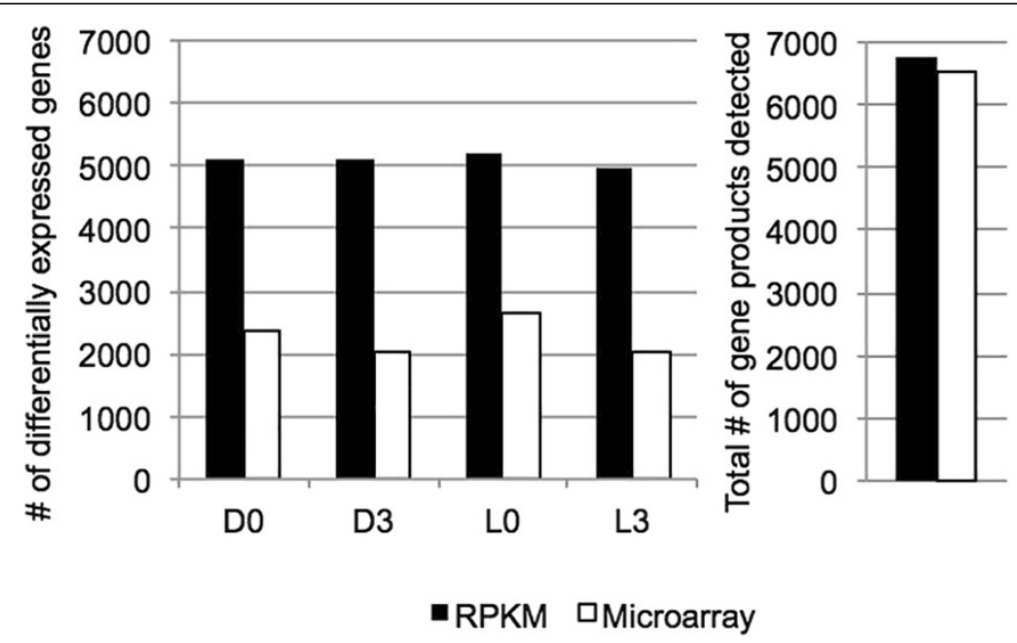

Figure 1 Comparison of gene expression at 4 time periods in Cyanothece 7822 grown under $\mathrm{N}_{2}$-fixing conditions in $12 \mathrm{~h}$ light-12 h dark measured through RNA-sequencing (RPKM) and by microarray. 


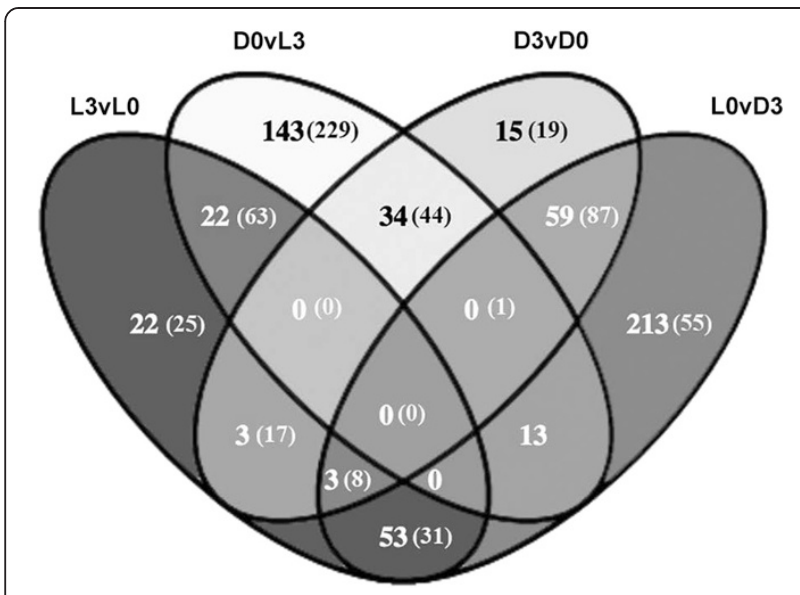

Figure 2 Venn diagram displaying the number of genes significantly up (bold lettering) and down (in parentheses) regulated $\geq \mathbf{2}$-fold across the various time points measured. Detailed listing of each group can be found in Additional file 5: Table S3.

each gene and (gene function) that is differentially regulated.

We concentrated our detailed analysis on the approximately 500 genes that comprise the major metabolic processes required for growth under diazotrophic, light-dark conditions, although the data for all genes is presented in the supplemental tables. During the light period, we observe high expression of genes related to bioenergetics, such as various $\mathrm{NADH}$-quinone oxidoreductases and $\mathrm{NAD}(\mathrm{P}) \mathrm{H}$ dehydrogenases, and $\mathrm{CO}_{2}$ fixation genes, such as carbon dioxide concentrating mechanism genes, ribulose-bisphosphate carboxylase and carbonic anhydrase. Other light-enhanced genes are those involved in the gluconeogenesis pathway, as well as the reductive pentose phosphate pathway as indicated by the presence of ribulose-phosphate 3-epimerase. Most predominant are genes responsible for encoding proteins involved in photosynthesis and glycogen biosynthesis. As the cell enters the dark phase, many of these genes become down regulated and genes involved in nitrogen fixation and butanoate metabolism (polyhydroxyalkonate synthesis, short-chain dehydrogenase, and acetyl-CoA acetyltransferase) become upregulated. In addition, genes of the TCA cycle are highly expressed and genes encoding proteins in aerobic respiration, such as cytochrome oxidases, Rieske (2Fe-2S) domain-containing proteins, and the uptake and bi-directional hydrogenase were strongly upregulated. Of course, these proteins are also needed to deplete the intracellular oxygen levels in the cytoplasm in order to protect the oxygen-sensitive nitrogenase enzymes. Much of the key metabolic information contained in Additional file 1: Table S1 and Additional file 2: Table S2 and described above is visualized readily in Figure 3.

Interestingly, the analysis indicated that the initiation of transcription of genes encoding some key metabolic functions was not restricted to the light or to the dark exclusively, as in Cyanothece 51142 [8,21]. For example, transcription of the $\mathrm{N}_{2}$-fixing enzymes, Cyan7822_3667 to 3684 , was upregulated in both the late light and the early dark. Similarly, many enzymes involved in heme and pigment biosynthesis and genes encoding phycobiliproteins were upregulated $>2 \mathrm{FC}$ at D3vsD0 and L0vsD3, indicating that expression was high throughout much of the dark period from three hours into the dark up to the end of the dark period. The genes for cytochrome oxidase and those involved in hydrogenase and uptake hydrogenase activity were transcribed in the light so that the function would be available once the nitrogenase proteins were available in the late light period (See Additional file 5: Table S3 and Additional file 6: Figure S3). In these cases, the genes were transcribed in preparation for the next important metabolic phase. All of these results are consistent with the physiological measurements [17], which demonstrated nitrogen fixation and hydrogen production just prior to the dark period.

Figure 3 emphasizes a few temporal points about transcription in Cyanothece 7822. First, transcription of important metabolic functions typically began in a light period prior to their maximum expression. Thus, nitrogen fixation genes transcription began in the light, but peaked at D3. The same was true for the TCA and much of the PPP cycle genes, as well as those involved with respiration. On the other hand, carbon fixation, starch metabolism and photosynthesis gene transcription began in the dark and peaked in the light. Therefore, the transcription was not as tightly synchronized as in Cyanothece 51142 (Figure 3).

Unexpectedly, there was an upregulation of a Cmr4 family CRISPR-associated RAMP gene (Cyan7822_6164). Upon further investigation, a significant number of CRISPR (clustered regulatory interspaced short palindromic repeats) associated genes were expressed in Cyanothece 7822 and were mainly located near either end of the large linear plasmid (Figure 4). We will discuss the CRISPR genes in greater detail in the antisense and CRISPR expression section.

\section{Protein expression}

One objective of this study was to compare and contrast protein expression with the transcriptional data in order to investigate in-phase and anti-phase abundance maxima [24]. We were able to generate a large protein dataset containing 1322 quantifiable proteins from the four time points. We again focused on proteins that were involved with key metabolic pathways as outlined in Additional file 2: Table S2. These proteins and their kinetics over the 4 time periods are shown in Additional file 7: Table S4. The experiment identified key proteins in each of the major functional categories that led to some 


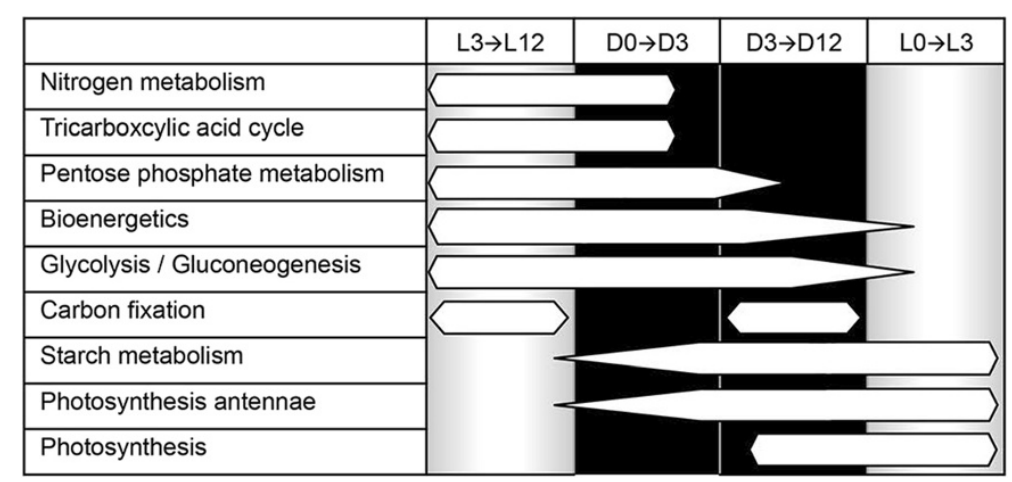

Figure 3 Diagram of differential gene expression in Cyanothece 7822 grown in 12-h light 12-h dark. Bar thickness is a qualitative measure of the mRNA levels at that time point. The two dark segments (early and late dark) are highlighted in black. The figure begins with the late light period (D0 vs L3) on the left to best represent the pattern of gene expression throughout the day to the early light period (L3 vs $L 0)$.

important insights into the regulation of metabolism in this unicellular diazotroph.

Proteins involved with cellular bioenergetics were mainly present in the dark. The highest levels of the cytochrome oxidase operon proteins, Cyan7822_47114713, were found in the dark, suggesting that this complex was used for respiration during the $\mathrm{N}_{2}$-fixing dark phase. Only one protein from the other complex, Cyan7822_4378, was identified and it was more constant throughout the experiment, although it dropped to the lowest levels at L3. The NADH dehydrogenase subunit 1, Cyan7822_2520, had levels some 2X higher at D3 than at the other times, also indicating that this protein was part of a dark-inducible complex.

As also seen in Cyanothece 51142, proteins encoded by the nitrogenase cluster genes were differentially expressed with a peak at D3 for all proteins except for NifD, which peaked at the end of the dark period. In a complementary fashion, the proteins involved in glutamate metabolism tended to be at higher levels in the late dark to early light.

Proteins involved in central metabolism (glycolysis, TCA cycle and PPP) demonstrated varied expression patterns (Figure 5). The figure displays the relative fold change of
mRNA levels with time in the upper two panels for each metabolic pathway along with the fold change in proteins levels with respect to time in the lower panel. The figure demonstrates that protein levels typically did not vary more than $25 \%$ throughout the daily cycle. However, in specific cases, the expression patterns demonstrated a light vs. dark periodicity. For example, most of the proteins for glycolysis were similar in the light and in the dark. Nonetheless, both copies of phosphofructokinase (Pfk) were expressed; one peaked in the light (Cyan7822_0159) and one peaked in the late dark period (Cyan7822_3033). Similarly, two Fba proteins were expressed, one in the light (Cyan7822_1659) and one in the dark (Cyan7822_1612). Neither protein was found exclusively in one time period, but the data suggested that Fba1 (Cyan7822_1612) was strongly upregulated in the late dark and early light, whereas Fba2 (Cyan7822_1659) was upregulated more in the late light and early dark periods. In both cases, the proteins were at reasonable levels throughout the light and the dark, although the results suggested that different Pfk and Fba proteins were expressed at different times for slightly different cellular needs. The TCA cycle proteins that were identified were slightly

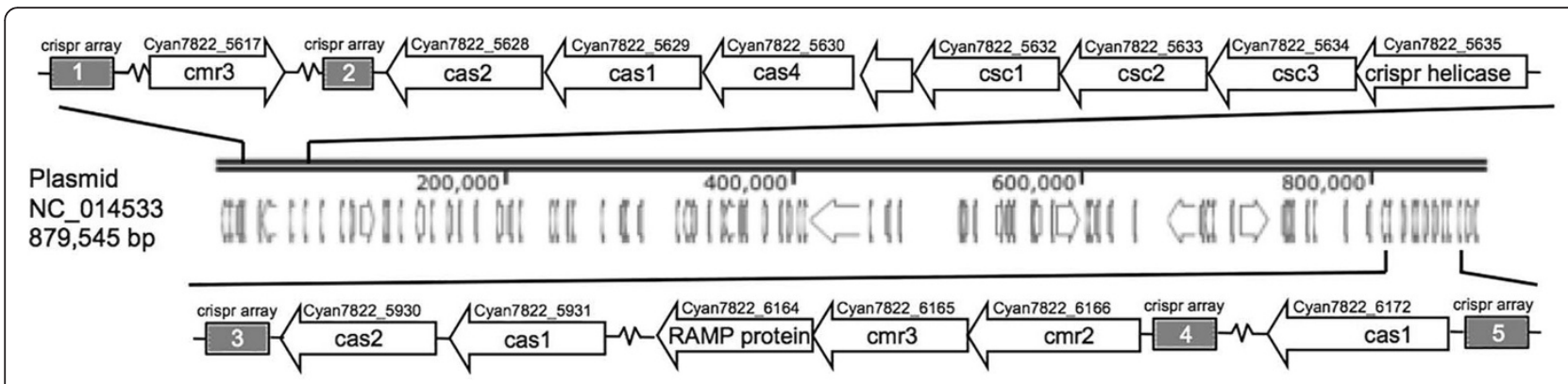

Figure 4 CRISPR genes on the large, linear plasmid (NC_014533) are located on both ends of the fragment and are highly transcribed in the late dark, early light period. Five repeat arrays were identified using CRISPRFinder and the CRISPRdb [22,23] and are represented as bars with the numbers $1-5$. 


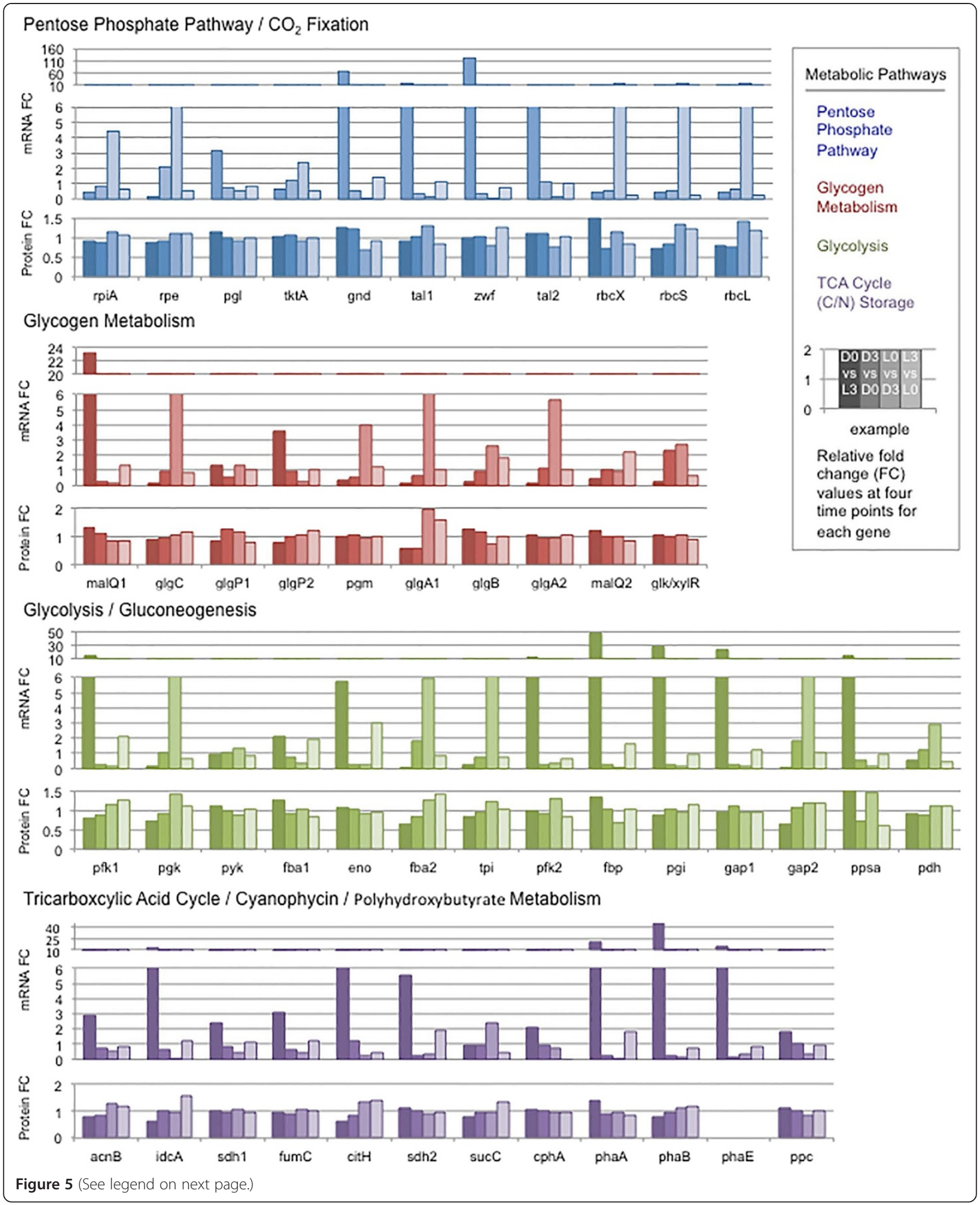


(See figure on previous page.)

Figure 5 The relative fold change expression levels of both mRNA and protein for $\mathbf{4 7}$ genes involved with central metabolism in Cyanothece $\mathbf{7 8 2 2}$ are shown. The $y$-axis represents the relative fold change in mRNA level or the relative fold change in protein level. The $x$-axis represents the four comparisons: D0 vs. L3 (mid-day to sunset); D3 vs. D0 (mid-night to sunset); D3 vs. L0 (mid-night to sunrise); and, L3 vs. L0 (sunrise to early morning). The figure is colored coded as follows for the protein-mRNA expression dynamics. PPP, blue; glycogen metabolism, red; glycolysis, green; and TCA, purple. Genes within each groups displayed are as follows: PPP and Carbon Fixation: Cyan7822_0018, rpiA; Cyan7822_0124, rpe; Cyan7822_0172, pgl; Cyan7822_1076, tktA; Cyan7822_3017, gnd; Cyan7822_4172, tal1; Cyan7822_4173, zwf; Cyan7822_5424, tal2; Cyan7822_2900, rbcX; Cyan7822_2901, rbcS; Cyan7822_2899, rbcL. Glycogen Metabolism: Cyan7822_1182, malQ1; Cyan7822_2049, glgC; Cyan7822_2294, glgP2; Cyan7822_2322, glgP1; Cyan7822_2491, pgm; Cyan7822_2570, glgA1; Cyan7822_2889, glgB; Cyan7822_4734, glgA2; Cyan7822_5145, malQ2; Cyan7822_5605, glk/xylR. Glycolysis: Cyan7822_0159, pfk1; Cyan7822_1455, pgk; Cyan7822_1523, pyk; Cyan7822_1612, fba1; Cyan7822_1633, eno; Cyan7822_1659, fba2 Cyan7822_1688, tpi; Cyan7822_3033, pfk2; Cyan7822_4171, fbp; Cyan7822_4412, pgi; Cyan7822_4423, gap1; Cyan7822_4638, gap2; Cyan7822_1627, ppsa; Cyan7822_2730, pdh. TCA and C/N Storage: Cyan7822_0752, acnB; Cyan7822_1400, idcA; Cyan7822_1551, sdh1; Cyan7822_2824, fumC; Cyan7822_3004, citH; Cyan7822_3071, sdh2; Cyan7822_3782, sucC; Cyan7822_2816, cphB; Cyan7822_1326, phaA; Cyan7822_1327, phaB; Cyan7822_1330, phaE; Cyan7822_2645, pcC.

higher in the light, indicating that this pathway is mostly involved with generating metabolites. Some of the proteins in the Pentose Phosphate Pathway, such as Rpi and Rpe, are also involved with $\mathrm{CO}_{2}$ fixation, and they were upregulated in the light. This paralled the induction of RuBP carboxylase, RbcLS, that was upregulated nearly 2-fold in the late-dark and early-light periods (Figure 5). On the other hand, PPP proteins were slightly upregulated in the early dark, and lowest in the late dark period, which suggested that a primary function was to provide reducing equivalents for $\mathrm{N}_{2}$ fixation during the early dark period. Importantly, glycogen synthase was strongly light regulated, appropriate for construction of the glycogen granules during carbon fixation. The two glycogen phosphorylase genes were both expressed as protein at all 4 time periods, but with slightly different kinetics: GlgP1 (Cyan7822_2322) had the highest abundance in the early light (L3), whereas GlgP2 (Cyan7822_2294) was highest in the late dark (L0). In a similar fashion, GlgA1 had a 3-fold higher level in the late-dark/early light than at other times, whereas GlgA2 was much more constant throughout the cycle.

\section{Relationship between transcription and translation Nitrogen fixation and photosynthesis}

The relationship between transcription and translation was one of the main objectives of this study and we again concentrated on the key metabolic categories. The nitrogenase gene cluster represents an important standard for such comparisons and is shown in Figure 6. These genes are all located in a large cluster and appear to be coordinately regulated at the transcriptional level, primarily in the dark. We were able to detect all of the proteins at all time points, except for nifZ and nifX. For

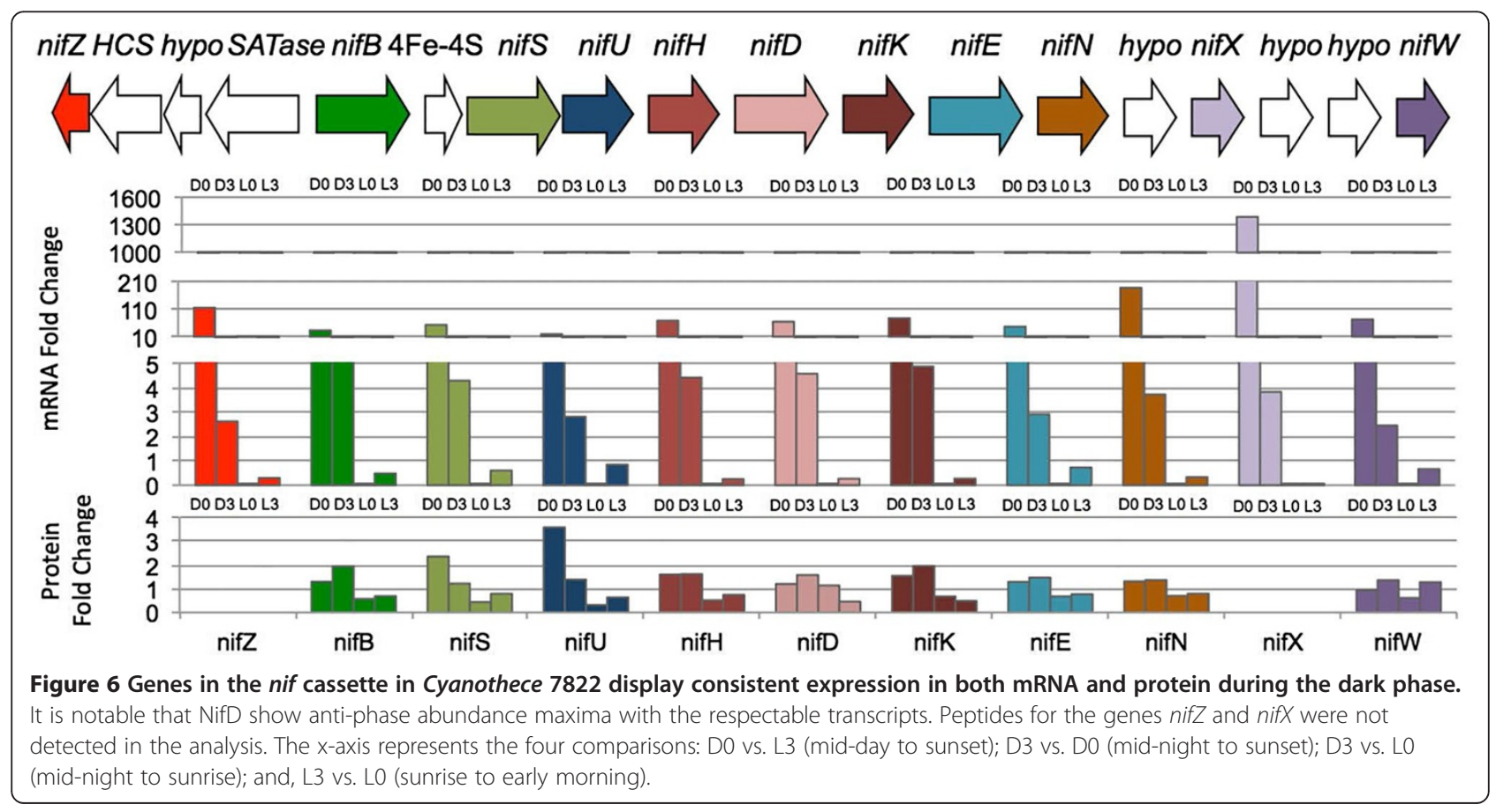


this group of genes, there was a great deal of correspondence between the timing of transcription and the appearance of the protein. The only one that was not in phase was nifD where the peak protein abundance lagged that of the mRNA. The protein abundance in the late light is high and quite similar to the mRNA pattern, thus establishing the nitrogenase cluster as an example of in-phase transcription/translation. The intensity of expression changes was substantial, with fold changes ranging from 10 to $>1000$ at the onset of the dark period. This emphasizes that the late light/early dark phase is the critical period for nitrogen fixation in Cyanothece 7822.

In regard to proteins involved in photosynthesis, not surprisingly most of photosystem I (PSI) and photosystem II (PSII) were higher in the light, whereas the cytb6/f complex and the ATPase synthase were fairly constant across the light periods. The photosynthetic antennae proteins that form the phycobilisomes were mostly light upregulated, except for the linker proteins that were somewhat higher in the dark. However, there were two interesting features that are also found in Cyanothece 51142: The PsaAB proteins were higher in the dark and one of the PsbA (D1) proteins (psbA4, Cyan7822_3753) was synthesized explicitly in the dark (some 2.5X higher at L0 (D12) than at the other time points). All sequenced cyanobacteria have multiple copies of $p s b A$ and some of these copies have mutations that likely render D1 incapable of binding the $\mathrm{Mn}_{4} \mathrm{CaO}_{5}$ metallocluster; thus, PSII complexes incorporating this altered DI would be incapable of $\mathrm{O}_{2}$ evolution [25]. We have noted that alternate copies of D1 are used by cyanobacteria under different environmental conditions, including high light, anoxic conditions or $\mathrm{N}_{2}$ fixation $[26,27]$. We have postulated that under $\mathrm{N}_{2}$-fixing conditions, the expression of such a modified D1 will permit a complete PSII complex to form, but one that cannot evolve $\mathrm{O}_{2}$. The amount of PsbA4 in the dark indicated that this is an important feature in Cyanothece 7822.

The Cyanothece 7822 genome contains 4 copies of the genes encoding the reaction center protein PsbA (also called D1). Three of these genes are virtually identical in sequence (psbA1 (Cyan7822_0979), psbA2 (Cyan7822_1825) and psbA3 (Cyan7822_2968)) and cannot be differentiated at the protein level. Figure 7 shows that the RNA levels for these 3 proteins are fairly constant over the 4 time periods in both the RNA-Seq (filled bars) and microarray experiments (empty bars) and that the composite protein level was also quite constant. However, psbA4 (Cyan7822_3753) mRNA was transcribed in the late light period and was high through the early dark. The protein was expressed in the late light and particularly strongly in the middle- to late-dark period. Thus, the $\mathrm{D} 1$ from $p s b A 4$ became an important component during $\mathrm{N}_{2}$ fixation when $\mathrm{O}_{2}$ levels should be at their lowest.

\section{Central metabolism}

We collected a quite complete set of data for the enzymes in central metabolism and identified a number of different patterns of transcription and translation (Figure 5). In glycolysis, it appeared clear that differential expression at the transcriptional level had little to do with the abundance of the proteins at various times throughout the diurnal cycle (compare the mRNA panels with the protein panels within each metabolic pathway). Differential transcription at D0 vs L3, D3 vs D0, L0 vs D3, and L3 vs L0 varied, ranging from modest changes in the mRNA ( $p y k)$ to extreme induction $(f b p$, $p f k$, and gap), whereas the observed protein abundance changed very little in most cases. The few exceptions were Fbp1 (Cyan7822_4171), Pgk (Cyan7822_1455), Gap2 (Cyan7822_4638) and Fba2 (Cyan7822_1659), as shown in Figure 5. For these genes, each of the mRNA transcripts and respective proteins increased and decreased more or less in concert with a slight lag for the increase in protein abundance. The discrepancy in the abundance changes of mRNA and protein suggests that post-translational regulatory mechanisms play an important role for central metabolism in Cyanothece 7822.

In the pentose phosphate pathway, the mRNA changes are far more striking than those of the proteins. This pathway is used in both the light and the dark and, although the transcripts of many proteins were most abundant in the late dark and early light, the protein levels remained high throughout much of the $24 \mathrm{~h}$ period. Interestingly, transcription of the genes for proteins also used for $\mathrm{CO}_{2}$ fixation was highest in the mid to late dark, thus preparing the cells for the onset of light-driven photosynthesis. This included the Rubisco genes for which the mRNA levels increased over 10-fold in the late dark and the protein levels were enhanced by about 2 -folld in the late dark and the early light. The genes for the TCA cycle were somewhat different, in that the main mRNA induction was typically in the daytime and the proteins seemed to be at slightly higher abundance in the light. Glycogen/starch metabolism is also represented by a number of different relationships where the proteins remained more stable than the mRNA levels would have indicated. The one instance where transcription and translation are in phase is the $c p h A$ gene that encodes the cyanophycin synthase. The expression patterns both are highest in the late light and progressively diminish during the dark into the early light. CphA is most needed during and after nitrogen fixation and so a high level at these times is appropriate.

\section{Protein expression across genomic elements}

Another objective was to analyze gene expression across the multiple elements that comprise this relatively complex genome. The genome of Cyanothece 7822 is the 


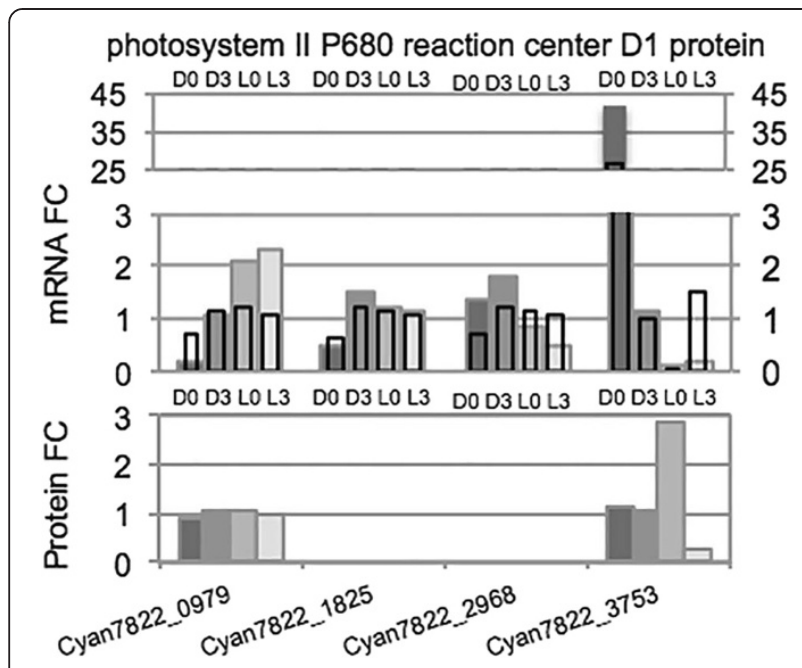

Figure 7 Expression levels for the $p s b A$ genes in Cyanothece 7822. Solid bars represent RNA-Seq and protein data and empty bars

represent microarray data. Due to their near identical sequences, D1 proteins psbA1 (Cyan7822_0979), psbA2 (Cyan7822_1825) and psbA3 (Cyan7822_2968) were quantitatively indistinguishable at the protein level. Microarray data points consisted of fold changes at D0vsL4 (instead of L3), D4vsD0 (instead of D3vsD0), LOvsD4, and $L 4 V s L O$

largest of the Cyanothece strains sequenced to date and is comprised of $6.1 \mathrm{Mb}$ circular chromosome, in addition to 6 extra-chromosomal elements, ranging in size from $879 \mathrm{~Kb}$ to $13 \mathrm{~Kb}$. An overview of how protein expression occurs from each genomic element is presented in Table 1. Over the $24 \mathrm{~h}$ time course, 1036 proteins were expressed with high quantitative reproducibility (see Methods section) from genes located on the main chromosome representing $18 \%$ of the presently annotated genes. However, a total of 1748 proteins from the circular chromosome were identified, at varying levels of quantitative accuracy, which accounted for

Table 1 Protein Expression in Cyanothece 7822 during $12 \mathrm{~h}, \mathrm{LD}, \mathrm{N}_{2}$-fixing growth [rounded to the nearest half percent]

\begin{tabular}{llll}
\hline Element & $\begin{array}{l}\text { Proteins } \\
\text { expressed }\end{array}$ & $\begin{array}{l}\text { Total genes } \\
\text { on element }\end{array}$ & $\begin{array}{l}\text { \% Genes } \\
\text { expressed }\end{array}$ \\
\hline chromosome NC_014501 6.1 MB & 1036 & 5663 & 18 \\
plasmid NC_014533 $879 \mathrm{~kb}$ & 53 & 595 & 9 \\
plasmid NC_014534 473 kb & 7 & 422 & 1.5 \\
plasmid NC_014502 $291 \mathrm{~kb}$ & 1 & 280 & 0.5 \\
plasmid NC_014503 $47 \mathrm{~kb}$ & 3 & 32 & 9 \\
plasmid NC_014504 $43 \mathrm{~kb}$ & 1 & 36 & 3 \\
plasmid NC_014535 $13 \mathrm{~kb}$ & 1 & 13 & 7.5 \\
\hline
\end{tabular}

representation of $31 \%$ of the total genes. All of the other genomic elements demonstrated some protein expression and the largest linear plasmid had 53 expressed proteins or $9 \%$ of the total ORFs on this plasmid. This genomic element encodes a number of very large genes and we obtained protein, of a variety of different functions, from 4 of these genes. Large expressed proteins included: Cyan7822_5813 with 1380 aa, which was at much higher levels in the early light. This protein may be involved in non-ribosomal peptide synthesis and could be involved with cyanophycin metabolism. Cyan7822_5889 encoded a 1211 aa protein that may be involved with Acyl-CoA synthesis and expression was highest in the late dark-early light periods. Cyan7822_5891 was among the largest polypeptides with 11,342 aa and expression was relatively unchanged throughout the day. This protein has many possible functional domains, but the largest component had homology to outer membrane adhesion-like proteins. Cyan7822_6000 with 5,687 aa was expressed with a peak in the dark and had homology to Concanavalin A-like lectin or glucanase. This protein does have a potential signal sequence at the $\mathrm{N}$ terminus and may be located in the cytoplasmic membrane, but the remainder of the protein does not have transmembrane segments. In addition, a protein encoded by the $43 \mathrm{~kb}$ plasmid was also large and upregulated in the light (Cyan7822_6655 with 1847 aa). The large proteins encoded by the chromosome cover many different functions as shown in Additional file 7: Table S4.

\section{Anti-sense and CRISPR expression}

The RNA-Seq experiment provided an opportunity to assess anti-sense RNA transcription in Cyanothece 7822. We know little about the overall importance of antisense RNA in cyanobacteria, but we can correlate global patterns in the light and dark and in regards to specific genomic elements. Anti-sense RNA was found throughout the genome, with sizes ranging from $\sim 50$ nt to over 500 nt. In addition, the anti-sense RNA density plot indicated that there were different levels of anti-sense throughout the light-dark cycle and that the amount of anti-sense RNA was lowest at D3. In analyzing the antisense as a function of genomic elements, the density was somewhat lower on plasmid 4, the $879 \mathrm{~kb}$ linear plasmid, and lowest on the $879 \mathrm{~kb}$ plasmid at D3. This relationship was verified by a more detailed statistical analysis as shown in Table 2. The main chromosome had a greater percentage of anti-sense RNAs than expected, whereas the $879 \mathrm{~kb}$ plasmid had many fewer anti-sense RNAs than expected, based on the total number of anti-sense RNAs detected. Although we have no direct indication that antisense-transcription regulates any specific cellular processes, the expression of antisense mRNA is not 
equal at the four time points measured. Such differences suggest that the antisense RNAs are modulated in response to the light-dark cycle and this may indicate a role in diurnal regulation.

One of the most interesting outcomes was the level of expression of specific genes found on this large linear plasmid 4 (Figure 4). Of the 595 genes, 15 or $2.5 \%$, are related to CRISPR activities in the cell. CRISPR genes have been found in many bacteria and provide them with adaptive sequence-specific nucleic acid restriction mechanisms that act as a defense against invading mobile genetic elements such as bacteriophages, invading plasmids, and transposons [28-33]. The presence of CRIPSR genes in Cyanothece is not unique to Cyanothece 7822 . In fact, all sequenced Cyanothece strains contain at least 10 annotated CRISPR-related genes (see Additional file 8: Table S5). For example, Cyanothece 51142 has 12 annotated CRISPR genes on its genome, all of which are located on the main circular chromosome (NC_010546) with none on the linear chromosome or the 4 plasmids. In similar fashion, Cyanothece sp. ATCC 51472 and Cyanothece sp. PCC 8801 also contain all of their 12 or 11 CRISPR genes, respectively, on their main chromosomes. Other Cyanothece strains, such as Cyanothece sp. PCC $8802,7425,7425$, and 7822 contain genes of this nature on both the main chromosome as well on their extrachromosomal plasmids. Cyanothece 7822 is most interesting because there is a total of 23 genes of CRISPR related functions, but only one gene is found on the main chromosome (NC 014501), whereas 15 are found on the large linear plasmid (NC_014533) and 7 on the second largest linear plasmid (NC_014534).

\section{Discussion}

\section{Comparison between Cyanothece strains}

A number of proteomic studies have now been performed on a series of Cyanothece strains, including the best studied, Cyanothece 51142. An intensive proteomic study of Cyanothece 51142 was performed [10], followed by a more detailed analysis of those proteins differentially expressed on cells grown under 15 different conditions [12]. This later study identified some 250 proteins (5\% of the predicted ORFs) that were differentially expressed. Their results for cells grown under nitrogenfixing conditions were similar to what was found here for Cyanothece 7822. One significant difference was that Cyanothece 51142 proteins from genes encoding nitrogenfixing and photosynthesis proteins were more highly synchronized to either the dark or light, respectively. On the other hand, the results for proteins involved in central metabolism were similar in both organisms.

A number of other cyanobacteria have been analyzed using RNA Seq including Synechococcus sp. PCC 7002 [34], Anabaena sp. PCC 7120 [35], Microcysits aeruginosa LE-3 [36] and Prochlorococcus MED4 [24]. The Prochlorococcus study was fundamentally the most similar to the current study, as it also analyzed proteomic data and obtained samples throughout a light-dark cycle. Prochlorococcus is the ocean's most abundant primary producer and must always live according to the natural diel cycle. Analysis of transcriptional patterns demonstrated a substantial amount of light (or dark) induced mRNA, but the protein levels sometimes differed sharply from that of the mRNA. Waldbauer et al. [24] analyzed time courses of paired mRNA-protein abundances for 312 proteins every $2 \mathrm{~h}$

Table 2 Antisense RNA vs Genome

\begin{tabular}{|c|c|c|c|c|c|c|c|c|c|c|c|}
\hline & & $\begin{array}{l}\text { chromosome } \\
\text { NC_014501 }\end{array}$ & NC_014533 & NC_014534 & NC_014502 & NC_014503 & NC_014504 & NC_014535 & Total & $\begin{array}{l}\text { Plasmid } \\
\text { count }\end{array}$ & $\%$ \\
\hline & $\begin{array}{l}\text { All } \\
\text { genes }\end{array}$ & 5330 & 558 & 362 & 246 & 29 & 31 & 10 & 6566 & 1236 & 18.8 \\
\hline \multirow[t]{3}{*}{ Do } & Count & 978 & 66 & 48 & 47 & 4 & 9 & 2 & 1155 & 177 & 15.3 \\
\hline & Expected & 937.2 & 98 & 64.1 & 43.3 & 5.1 & 5.4 & 1.8 & & & \\
\hline & p-value & $6.40 \mathrm{E}-04^{*}$ & $1.6 \mathrm{E}-04^{*}$ & $0.026^{*}$ & 0.51 & .591 & 0.097 & 0.33 & & & \\
\hline \multirow[t]{3}{*}{ D3 } & Count & 921 & 55 & 58 & 42 & 2 & 7 & 3 & 1088 & 167 & 15.3 \\
\hline & Expected & 883 & 92.5 & 60.2 & 40.7 & 4.7 & 5.1 & 1.7 & & & \\
\hline & p-value & $9.20 \mathrm{E}-04^{*}$ & $5.80 \mathrm{E}-06^{*}$ & 0.74 & 0.82 & 0.18 & 0.37 & 0.26 & & & \\
\hline \multirow[t]{3}{*}{ LO } & Count & 994 & 63 & 66 & 41 & 2 & 5 & 2 & 1173 & 179 & 15.3 \\
\hline & Expected & 951.7 & 99.7 & 64.9 & 44.1 & 5.2 & 5.5 & 1.8 & & & \\
\hline & p-value & 4.30E-04* & $2.30 \mathrm{E}-05^{*}$ & 0.88 & 0.6 & 0.12 & 0.8 & 0.9 & & & \\
\hline \multirow[t]{3}{*}{ L3 } & Count & 1069 & 71 & 63 & 36 & 3 & 7 & 2 & 1252 & 183 & 14.6 \\
\hline & Expected & 1016.4 & 105.9 & 69.4 & 46.8 & 5.5 & 6,1 & 1.9 & & & \\
\hline & p-value & 1.30E-05* & 7.20E-05* & 0.39 & 0.068 & 0.21 & 0.67 & 0.39 & & & \\
\hline
\end{tabular}

* Indicates significance with $\mathrm{p}<0.25$. 
that represents about $16 \%$ of the 1955 ORFs in the genome. This is similar to the overall percentage from Cyanothece 7822 that we studied. In the case of both Cyanothece strains analyzed in detail, the closest convergence for mRNA and protein were for the nitrogenase cluster in the night-this seems to be critical, since the Nif complex is readily degraded by $\mathrm{O}_{2}$ that is most prevalent in the light.

\section{Future potential for study in Cyanothece 7822}

Another benefit of having paired mRNA and protein data is the ability to understand better the relationship of transcription and translation of metabolic pathways that generate intermediates for various targeted products. For example, Cyanothece 7822 has potential to be engineered for butanol production. Cyanothece 7822 possesses at least two pathways for butanol production (Figure 8), via isoleucine biosynthesis and via the crotonyl-CoA pathway. Isoleucine synthesis in Cyanothece 7822 is similar to that in Cyanothece 51142 and occurs through an alternate citramalate pathway similar to anaerobic bacteria and archaea [37]. Citramalate is generated through a condensation reaction of pyruvate and acetyl-CoA catalyzed by $\mathrm{CimA}$ then to 2-ketobutyrate and eventually isoleucine. The catalytic actions of the native LeuABCD that convert 2-ketobutyrate to 2-ketovalerate, along with the additional actions of a heterologous 2ketoacid-decarboxylase (KdcA) and a dehydrogenation reaction, would theoretically allow butanol production. Generating butanol via the crotonyl-CoA pathway would require inhibition of $\mathrm{PHB}$ synthesis along with the introduction of two heterologous genes, 3-hydroxybutyryl dehydrogenase (crt) and trans-2-enoyl-CoA reductase (ter). Since Cyanothece is missing these last two genes in the biosynthesis pathway, as well as a 2-keto decarboxylase, it is proposed that the introduction of these 3 genes into Cyanothece 7822 would result in the phototrophic production of butanol. This is similar to genetic manipulations in Synechococcus elongatus PCC 7942 that led to the production of butanol [38] and isobutyraldehyde [39]. In both these pathways, a variety of comparative patterns can be seen, including inphase and anti-phase. These results indicate that many different relationships occur between sense and antisense transcription and translation in Cyanothece 7822 and that targeting this strain to produce a specific metabolite will require in depth analysis of expression patterns, paying careful attention to the interplay between sense and antisense transcription and their relationship to translation levels.

While techniques to genetically modify Cyanothece 7822 to carry out these modifications exist, advancements in commandeering the cas 9 CRISPR enzyme for genome editing in bacteria could provide an alternative, and possibly, more efficient method [40,41]. Cyanothece 7822 has a cluster of CRISPR genes on the extra-chromosomal plasmids. These genes display high levels of transcription, yet of the 23 genes annotated as being involved in CRISPR activities that are transcriptionally active, only one was observed in the protein population. While it is unclear what role these active genes are playing in the cell, their activity suggests that the introduction of cas9-based editing tools may be a viable new option that should be pursued further. In addition, it is notable that CRISPR-like genes are located on the ends of the large plasmid, a fact that allows the speculation that the pressure for maintenance of these extra-chromosomal plasmids is connected to their presumptive anti-viral activity. The reason for the complexity of the Cyanothece genomes [3] is unknown, but the possibility of horizontal gene transfer of DNA elements, followed by stabilization, is one possibility. The importance of specific gene functions on these introduced elements can be the reason that they have been preserved. We need to study gene expression in Cyanothece 7822 under many different conditions in order to test this general hypothesis and know exactly how these extra chromosomal elements are providing a benefit for the cell and it will be interesting to see if cyanophage that infect any of the Cyanothece strains can be identified in the future.

\section{Conclusions}

An understanding of bacterial complexity requires the analysis of the full complement of proteins and the way they are regulated. We have begun this process in Cyanothece 7822 by analyzing transcription and translation across a $24 \mathrm{~h}$ diurnal period under nitrogen-fixing conditions. Overall, the results indicate that: (1) RNA Seq and microarray data provide similar information on mRNA transcription, although RNA Seq provides more coverage and higher sensitivity; (2) Although we can obtain complete coverage at the mRNA level, the number of proteins identified represents about $25 \%$ of all possible gene products; (3) Differential expression at the mRNA and protein level can follow the same general pattern or can be quite different. Strongly up- or down-regulated genes at the transcriptional level are no guarantee that the proteins will behave similarly; (4) Nonetheless, many proteins involved in key metabolic functions demonstrated expression to a greater extent in either the light or the dark, depending upon the need for that function. The dynamic range of the protein levels under these conditions is typically far less than that shown at the messenger RNA level, implying that statements relating to absolute levels of induction/repression at the transcriptional level may not be important at the protein level.

\section{Methods}

\section{Cell cultivation}

Cyanothece 7822 was grown in modified BG-11 medium [16] with the nitrate removed. For the RNA-seq 


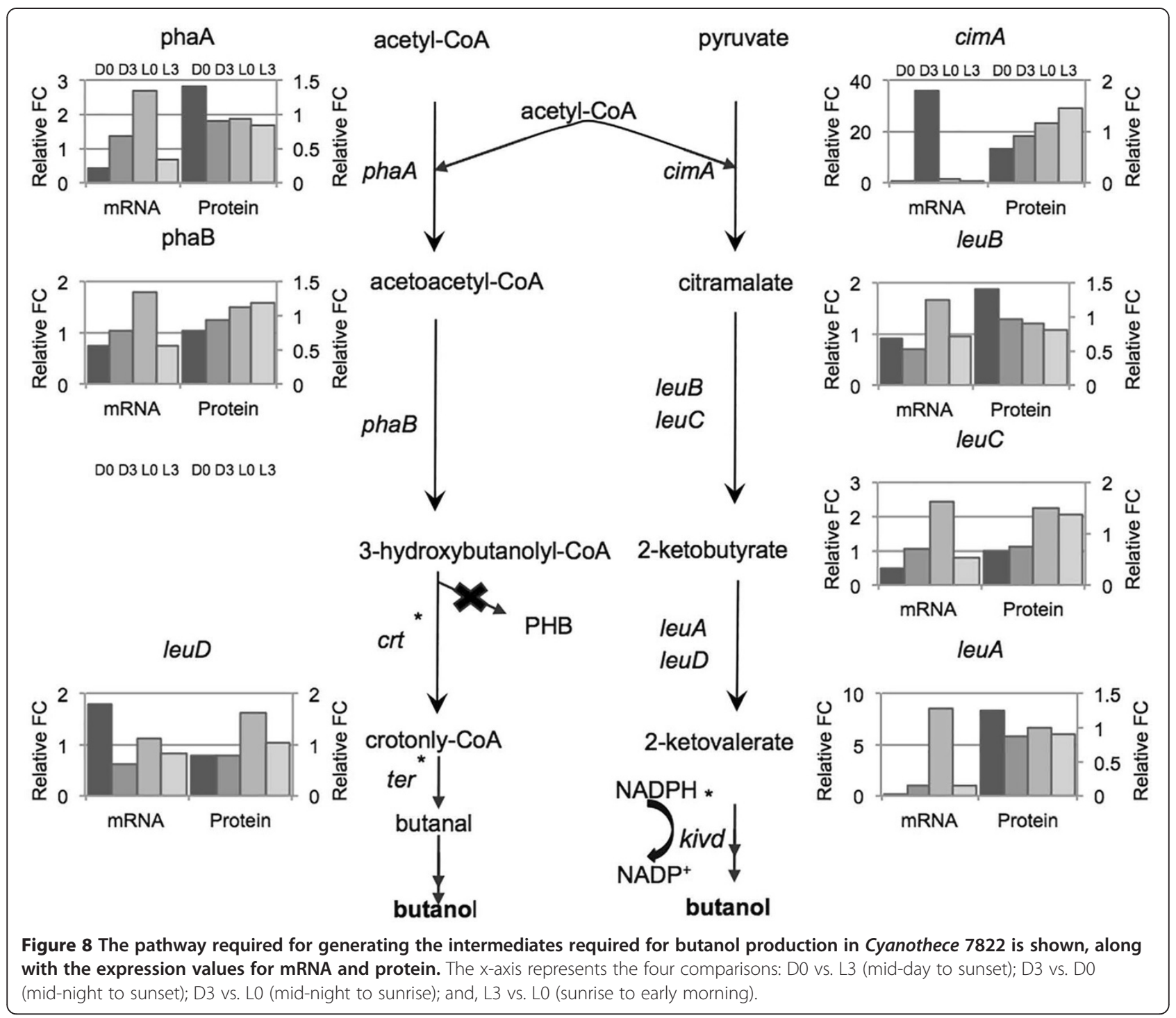

analysis, cultures were grown in $100 \mathrm{ml}$ volumes in 250 $\mathrm{ml}$ Erlenmeyer flasks at $30^{\circ} \mathrm{C}$ with shaking at $125 \mathrm{rpm}$ and $30-40 \mu \mathrm{mol}$ photons $\mathrm{m}^{-2} \mathrm{~s}^{-1}$ of light from cool white fluorescent bulbs; or 2 . For the microarray analysis, cultures were grown in airlift bioreactors (6-liter BioFlo 3000; New Brunswick Scientific, Edison, NJ) illuminated by two light-emitting-diode panels using alternating arrays of orange $(640 \mathrm{~nm})$ and red $(680 \mathrm{~nm})$ light, providing an intensity of $\sim 100 \mu \mathrm{mol}$ photons $\mathrm{m}^{-2}$ $\mathrm{s}^{-1}$ at $30^{\circ} \mathrm{C}$. Experimental conditions consisted of $12-\mathrm{h}$ light-12-h dark cycles for 3 to 4 days. Stock cultures grown in continuous light were used to inoculate experimental flasks at a density of approximately $1 \times 10^{6}$ cells $\mathrm{ml}^{-1}$. For all RNA and protein collection cellular material was cooled to $\sim 4^{\circ} \mathrm{C}$ with a refrigerated centrifuge and the material was stored on ice.

\section{Extraction and processing of $\mathrm{mRNA}$}

For RNA-Seq analysis, cellular material from each time point was cooled on ice to limit metabolic changes, kept at $4^{\circ} \mathrm{C}$, and transported to the Environmental Molecular Sciences Laboratory at Pacific Northwest National Labs in Richland, WA. The RNA was extracted using Invitrogen TRIzol ${ }^{\circ}$ Reagent (cat\#15596018), followed by genomic DNA removal and cleaning using the Qiagen RNase-Free DNase Set kit (cat\#79254) and the Qiagen Mini RNeasy ${ }^{\text {rm }}$ kit (cat\#74104). The Agilent 2100 Bioanalyzer was used to assess the integrity of the RNA samples. Only RNA samples having RNA Integrity Number $\geq 8$ were used for RNA Seq. Deep RNA sequencing was performed on the 5500xl SOLiD ${ }^{\mathrm{mm}}$ sequencing platform (Life Technologies). Applied Biosystems SOLiD $^{\text {tw }}$ Total RNA-Seq kit (cat\# 4445374) was used to 
generate the template cDNA library following the manufacturer recommended protocol. Briefly, the mRNA was fragmented using chemical hydrolysis followed by ligation with strand-specific adapters and reverse transcription to generate cDNA. Fragments of cDNA between 150 to 250 bp were subsequently isolated by electrophoresis in $6 \%$ urea-TBE acrylamide gel. The gel-isolated cDNA fragments went through 15 amplification cycles to produce enough templates for the SOLiD ${ }^{\mathrm{TM}} \mathrm{EZ}$ Bead ${ }^{\mathrm{TM}}$ system (Life Technologies), which was used to perform emulsion clonal bead amplification to generate a template bead library for ligation-base sequencing using the SOLiD ${ }^{\text {sm }} 5500 x 1$ instruments. Samples were analyzed at the Environmental Molecular Sciences Laboratory (EMSL) at Pacific Northwest National Laboratory (Richland, WA, USA). At times, cells were transported at $4^{\circ} \mathrm{C}$ and briefly thawed at room temperature prior to analysis.

For microarray analysis, RNA was extracted immediately after harvesting cells at each time point (D0, D4, L0 and L4) using Tri-Reagent ${ }^{\odot}$ (Sigma-Aldrich cat\#T9424) according to the manufacturer's protocol. Samples were resuspended in Tri-Reagent and washed with glass beads at temperature below $0^{\circ} \mathrm{C}$ followed by phase separation using 1-bromo-3-chloropropane. The time points were slightly different than in the RNA-Seq experiment, but we have shown that there is very little difference in expression levels between 3 and $4 \mathrm{~h}$ in either the light or dark in Cyanothece 7822. RNA was precipitated with isopropanol, and RNA clean-up kit-5 columns from Zymo Research Corporation (Orange, CA) were used to remove contamination. The hybridization control consisted of a mixed sample that contained equal amounts of RNA from each time point. Four biological and two technical replicates were included in analysis. For each microarray, $7 \mu \mathrm{g}$ RNA was used $(3.5 \mu \mathrm{g}$ sample plus $3.5 \mu \mathrm{g}$ control). Total RNA was labeled with either cyanine- 5 or cyanine- 3 by using a ULS RNA fluorescent-labeling kit from Kreatech Biotechnology (Amsterdam, The Netherlands) according to the manufacturer's protocol. The labeled material was passed through Zymo RNA clean-up kit-5 columns (Zymo Research Corporation, Orange, CA) to remove unincorporated label and eluted in 15 to $20 \mu \mathrm{l}$ of RNase-free water. The concentration of labeled total RNA and label incorporation was determined on a Nanodrop-1000 spectrophotometer (Wilmington, DE). All of the labeling and post labeling procedures were conducted in an ozone-free enclosure to ensure the integrity of the label. The microarray platform consisted of 6538 ORFs based upon the Cyanothece genome sequence. The 60-mers appropriate for each gene were determined by MoGene and hybridization; scanning, and initial data analysis was conducted at MoGene (St. Louis, MO).

\section{Generation of RNA Seq data-files}

The 50-base short read sequences produced by the $5500 X \mathrm{~L} \mathrm{SOLiD}^{\mathrm{TM}}$ sequencers were mapped to the Cyanothece 7822 reference genome (http://www.ncbi.nlm.nih. gov/genome/) using the default settings in the Life Technologies software provided for the SOLiD sequencers (LifeScope ver. 2.5 for the SOLiD 5500xl). LifeScope Whole Transcriptome analysis generated the following files: 1) a BAM file containing the sequence of every mapped read and its mapped location; 2) pairs of *.wig files (one for each strand on the chromosome) with the mapped counts at each base position; and 3) a gene counts file, with base counts summed to a single value across the entire gene length and RPKM values given for each gene.

\section{Semiquantitative RT-PCR}

RNA was treated with DNase I (Invitrogen, Carlsbad, CA) for $1 \mathrm{~h}$ at $37^{\circ} \mathrm{C}$ and followed by DNase I treatment for 30 minutes at $37^{\circ} \mathrm{C}$. Reverse transcription (RT) was performed by using Superscript II (Invitrogen, Carlsbad, $\mathrm{CA}$ ) with random primers following the manufacturer's instructions. PCR was carried out at $94^{\circ} \mathrm{C}$ for $1 \mathrm{~min}, 30$ cycles of $94^{\circ} \mathrm{C}$ for $30 \mathrm{~s}$ and $53^{\circ} \mathrm{C}$ for $30 \mathrm{~s}$, and $72^{\circ} \mathrm{C}$ for $30 \mathrm{~s}$ to amplify regions of the genes hoxH, hupL nifD, $p s b A$, and $r p n B$. PCR was carried out to amplify regions of the genes for $r n p B$ (22 cycles), hoxH (32 cycles), hupL (28 cycles), nifD (32 cycles), and $p s b A$ (30 cycles). The $r p n B$ transcript abundance was used as a control, since results from the microarray analysis indicated that the transcript level for this gene was unchanged under these growth conditions across each time point. The primers for each transcript were as follows: rnpBF 5'-CGTGAGGATAGTGCCACAGA3', rnpBR5'- AAACGGGACCGGTAAAAGAC-3,' hoxHF 5'GCTGAAGCCGGAATTAACAA-3;' hoxHR 5'-ATTTGTA GCGGCATTTGTCC-3,' hupLF 5'-AACGGTAAACCGATCAAACG-3', hupLR 5'-CGGATGGGTCTTGATATTGG-3, nifDF 5'-ATTTCCAAGAACGCGACATC-3, nifDR 5'-TC ACGAACAGCATCGTTAGC-3,' psbAF 5'-CCCACCCTTC TGACAGCTAC-3, psbAR 5'CTAACTGGTAAGGGCCAC $C A-3$ '.

\section{Protein sample preparation}

Cell suspensions were centrifuged for $30 \mathrm{~min}$ at $1000 \times \mathrm{g}$ at $4^{\circ} \mathrm{C}$, reduced to $0.5 \mathrm{~mL}$ volume, and then washed at $4^{\circ} \mathrm{C}$ with $2 \mathrm{~mL}$ of $\mathrm{NH}_{4} \mathrm{HCO}_{3}, \mathrm{pH} 7.8$, three times. To the final pelleted cells, $0.5 \mathrm{~mL}$ of lysis buffer ( $8 \mathrm{M}$ Urea in $\mathrm{NH}_{4} \mathrm{HCO}_{3}, \mathrm{pH} 7.8$ ) was added and they were homogenized for $5 \mathrm{~min}$ using a Qiagen TissueRuptor followed by a BCA assay (Pierce) to determine protein concentration. Protein samples were incubated in $8 \mathrm{M}$ Urea, $5 \mathrm{mM}$ dithiothreitol at $56^{\circ} \mathrm{C}$ for $45 \mathrm{~min}$ with shaking at $800 \mathrm{rpm}$ in Thermomixer R (Eppendorf). Alkylation was performed with $20 \mathrm{mM}$ iodoacetamide at $37^{\circ} \mathrm{C}$ in dark with shaking (800 rpm in Thermomixer) followed by and 8 fold dilution with nanopure water 
containing $1 \mathrm{mM} \mathrm{CaCl}$. Tryptic digest with Sequencing Grade Modified Trypsin (Promega) was performed at 1:50 enzyme-to-substrate ratio for $4 \mathrm{~h}$ at $37^{\circ} \mathrm{C}$. The digested samples were then acidified with $10 \%$ trifluoroacetic acid to $\sim \mathrm{pH} 3$ and 5\% acetonitrile was added to the digested samples prior to desalting. SPE C-18 columns (SUPELCO Discovery) were used for clean-up of the resultant peptide mixture, and samples were concentrated down using the SpeedVac SC250 Express (ThermoSavant) followed by BCA assay to determine final peptide concentration.

\section{ITRAQ labeling and HPLC fractionation}

Isobaric labeling of captured peptides to achieve relative quantification using four-plex iTRAQ ${ }^{\mathrm{TM}}$ reagents was performed according to the manufacturer's instructions ( $A B$ Sciex, Foster City, CA). For iTRAQ ${ }^{\mathrm{Tm}}$ labeling, $30 \mu \mathrm{g}$ of peptide for each time point was resuspended in $10.0 \mu \mathrm{L}$ of dissolution Buffer (500 mM triethylammonium bicarbonate) mixed with the individual iTRAQ ${ }^{\mathrm{TM}}$ reagent and incubated at RT for $1 \mathrm{~h}$. The reaction was stopped and unreacted iTRAQ reagents were hydrolyzed by adding $150 \mu \mathrm{L}$ of $\mathrm{H}_{2} \mathrm{O}$ and incubation at RT for $30 \mathrm{~min}$. Contents of each iTRAQ reagent-labeled sample was then pooled, followed by concentration down to $100 \mu \mathrm{L}$ prior to HPLC fractionation.

Labeled peptide samples were separated at flow rate at $0.5 \mathrm{~mL} / \mathrm{min}$ on a reverse phase Waters XBridge C18 column $(250 \mathrm{~mm} \times 4.6 \mathrm{~mm}$ column containing $5-\mu \mathrm{m}$ particles, and a $4.6 \mathrm{~mm} \times 20 \mathrm{~mm}$ guard column) using an Agilent 1200 HPLC System equipped with a quaternary pump, degasser, diode array detector, Peltier-cooled auto-sampler and fraction collector (both set at $4^{\circ} \mathrm{C}$ ). $120 \mu \mathrm{g}$ of labeled tryptic peptides was suspended in buffer A (10 mM triethylammonium bicarbonate, $\mathrm{pH} 7.5)$ and loaded onto the column. After the sample loading, the $\mathrm{C} 18$ column was washed for $35 \mathrm{~min}$ with solvent A, before applying the LC gradient. The LC gradient started with a linear increase of solvent A to 10\% B (10 mM triethylammonium bicarbonate, $\mathrm{pH} 7.5,90 \%$ acetonitrile) for $10 \mathrm{~min}$, then linearly increased to $20 \% \mathrm{~B}$ at $15 \mathrm{~min}$, $30 \mathrm{~min}$ to $30 \% \mathrm{~B}, 15 \mathrm{~min}$ to $35 \% \mathrm{~B}, 10 \mathrm{~min}$ to $45 \% \mathrm{~B}$ and another $10 \mathrm{~min}$ to $100 \%$ solvent B. Using an automated fraction collector, 96 fractions were collected for each sample, lyophilized and reconstituted into 12 fractions prior to LC-MS/MS analysis. These 12 final fractions were concatenated by combining each 8 fractions that were 12 fractions apart.

\section{LC-MS/MS analysis}

All iTRAQ ${ }^{\text {im }}$-labeled fractions were analyzed by LC-MS/ MS. Each sample was loaded onto a homemade $65 \mathrm{~cm} \times$ $75 \mathrm{~mm}$ i.d. reversed-phase capillary column using 3-mm C18 particles (Phenomenex, Torrance, CA, USA). The HPLC system consisted of a custom configuration of 100- ml Isco Model 100DM syringe pumps (Isco, Lincoln, NE, USA), two-position Valco valves (Valco Instruments Co., Houston, TX, USA), and a PAL autosampler (Leap Technologies, Carrboro, NC, USA) that allowed fully automated sample analysis across four HPLC columns [42]. The system was operated at a constant pressure of 10,000 psi over $3 \mathrm{~h}$ with an exponential gradient starting with $100 \%$ of mobile phase A $(0.1 \%(\mathrm{v} / \mathrm{v})$ formic acid in water) to $60 \%(\mathrm{v} / \mathrm{v})$ of mobile phase B $(0.1 \%(\mathrm{v} / \mathrm{v})$ formic acid in acetonitrile). MS analysis was performed on a Thermo Scientific LTQ-Orbitrap Velos mass spectrometer (Thermo Scientific, San Jose, CA, USA) coupled with an electrospray ionization interface using home-made 150-mm o.d. $\times$ 20-mm i.d. chemically etched electrospray emitters [43]. Full MS spectra were recorded at resolution of $100 \mathrm{~K}(\mathrm{~m} / \mathrm{z}$ 400) over the range of $\mathrm{m} / \mathrm{z} 400-2000$ with an automated gain control (AGC) value of $1 \times 10^{6}$. MS/MS was performed in the data-dependent mode with an AGC target value of $3 \times 10^{4}$. The most abundant 10 parent ions were selected for MS/MS using high-energy collision dissociation with a normalized collision energy setting of 45. Precursor ion activation was performed with an isolation width of $2 \mathrm{Da}$, a minimal intensity of 500 counts, and an activation time of $10 \mathrm{~ms}$.

\section{Data analysis}

LC-MS/MS raw data were converted into data files using Bioworks Cluster 3.2 (Thermo Fisher Scientific, Cambridge, MA, USA), and MSGF+ algorithm [44] was used to search MS/MS spectra against the Cyanothece 7822 sequence (NCBI 2011-02-28). The key search parameters used were 20 ppm tolerance for precursor ion masses, partial tryptic search, dynamic oxidation of methionine (15.9949 Da), static IAA alkylation on cysteine $(57.0215 \mathrm{Da})$, and static iTRAQ modification of lysine and N-termini $(+144.1021$ $\mathrm{Da})$. The decoy data base searching methodology $[45,46]$ was used to control the false discovery rate at the unique peptide level to $\sim 0.1 \%$ [47]. For quantification purposes, peptide reporter ion intensities were captured across all channels and compared by calculating the summed peptide intensity protein ratio between $\mathrm{L} 3, \mathrm{D} 0$, and D3 versus L0. Protein values with a correlation of $>0.5$ between biological replicates were retained. Certain proteins, such as RbcLS, with a correlation of 0.46 were included in our analysis. These highly abundant proteins fluctuated among our biological replicates, but always had the same periodicity and were important for the overall analysis. Protein values were then central tendency normalized using the program DAnTE [48] for direct comparison against mRNA values.

\section{Additional files}

Additional file 1: Table S1. mRNA Summary 
Additional file 2: Table S2. Functional mRNA Expression.

Additional file 3: Figure S1. Four scatter plots to illustrate the correlation between the RNA Seq method (voom, RPKM) and the microarray method in terms of log2 fold changes. A) Illustrates the correlation of common significant differentially expressed genes (at FDR $<0.05$ ) between the RNA Seq method (RPKM) and the microarray method for D0 vs L0 comparison on $x$-axis and $y$-axis respectively. The Spearman's correlation coefficient for this comparison is 0.846 . B) Illustrates the correlation of the common significant differentially expressed genes (at FDR $<0.05$ ) between the RNA Seq method (voom) and the microarray method for a D0 vs LO comparison on the $x$-axis and $y$-axis, respectively. The Spearman's correlation coefficient for this comparison is 0.877 . C) llustrates the correlation of all genes between the RNA Seq method (RPKM) and the microarray method for the DO $\vee$ LO comparison on the $x$-axis and $y$-axis, respectively. The Spearman's correlation coefficient for this comparison is $0.667 \mathrm{D}$ ) Illustrates the correlation of all genes between the RNA-Seq method (voom) and the microarray method for the D0 vs. L0 comparison on the $x$-axis and the $y$-axis, respectively.

Additional file 4: Figure S2. Comparison heat map showing up (red) and down (green) regulation of the major metabolic genes in Cyanothece 7822 for the microarray platform and the RNA-seq method.

Additional file 5: Table S3. Total mRNA Expression.

Additional file 6: Figure $\mathbf{S 3}$. Acrylamide gel patterns of key metabolic genes in Cyanothece 7822 critical for nitrogen fixation and photosynthesis measured by reverse transcriptase PCR. The results correlated very closely to that of the RNA Seq and microarray experiments.

Additional file 7: Table S4. Protein

Additional file 8: Table S5. CRISPR.

\section{Abbreviation}

PHB: Polyhydroxybutyrate.

\section{Competing interests}

The authors declare that they have no competing interests.

\section{Authors' contributions}

DW grew cultures, analyzed RNA-Seq and protein data, prepared figures and supplemental tables, and wrote the initial draft of manuscript. XZ grew cultures, analyzed microarray data and prepared figures. DW and XZ are co-first authors. MM, RT and GO performed the RNA-Seq experiment and performed initial analysis. JJ and MG performed proteomics experiments and initial analysis. KB and JT performed initial statistical analysis on all data. HM performed a detailed bioinformatics analysis on RNA-Seq data, especially anti-sense RNA transcription. RS contributed to proteomic instrumental design and execution. LS conceived the study, participated in the design and analysis of the various components, and wrote the final manuscript. All authors read and approved the final manuscript.

\section{Acknowledgements}

Part of this research was performed at the Environmental Molecular Sciences Laboratory (EMSL). EMSL is a U.S. Department of Energy (DOE) Office of Biological and Environmental Research national scientific user facility on the Pacific Northwest National Laboratory (PNNL) campus. PNNL is a multiprogram national laboratory operated by Battelle for the DOE under contract DE-AC05-76RL01830. This work was supported by a grant from the Office of Science (BER), U. S. Department of Energy to Drs. Pakrasi and Sherman.

\section{Author details}

'Department of Biological Sciences, Purdue University, West Lafayette, IN, USA ${ }^{2}$ Environmental Molecular Sciences Laboratory, Pacific Northwest National Laboratory, Richland, WA, USA. ${ }^{3}$ Biological Sciences Division, Pacific Northwest National Laboratory, Richland, WA, USA. ${ }^{4}$ Bioinformatics Core, Purdue University, West Lafayette, IN, USA

Received: 7 August 2014 Accepted: 16 December 2014

Published: 29 December 2014

\section{References}

1. Min HT, Sherman LA: Genetic transformation and mutagenesis via singlestranded dna in the unicellular, diazotrophic cyanobacteria of the genus Cyanothece. Appl Environ Microbiol 2010, 76(22):7641-7645.

2. Zhang $X$, Sherman DM, Sherman LA: The Uptake Hydrogenase in the Unicellular Diazotrophic Cyanobacterium Cyanothece sp. Strain PCC 7822 Protects Nitrogenase from Oxygen Toxicity. J Bacteriol 2014, 196(4):840-849.

3. Bandyopadhyay A, Elvitigala T, Welsh E, Stockel J, Liberton M, Min H, Sherman LA, Pakrasi HB: Novel metabolic attributes of the genus Cyanothece, comprising a group of unicellular nitrogen-fixing Cyanothece. Mbio 2011, 2(5). doi:10.1128/mBio.00214-11.

4. Bandyopadhyay A, Stockel J, Min H, Sherman LA, Pakrasi HB: High rates of photobiological $\mathrm{H} 2$ production by a cyanobacterium under aerobic conditions. Nat Commun 2010, 1:139.

5. Elvitigala T, Stockel J, Ghosh BK, Pakrasi HB: Effect of continuous light on diurnal rhythms in Cyanothece sp. ATCC 51142. BMC Genomics 2009, 10:226.

6. Singh AK, Elvitigala T, Cameron JC, Ghosh BK, Bhattacharyya-Pakrasi M, Pakrasi HB: Integrative analysis of large scale expression profiles reveals core transcriptional response and coordination between multiple cellular processes in a cyanobacterium. BMC Syst Biol 2010, 4:105.

7. Stockel J, Welsh EA, Liberton M, Kunnvakkam R, Aurora R, Pakrasi HB: Global transcriptomic analysis of Cyanothece 51142 reveals robust diurnal oscillation of central metabolic processes. Proc Natl Acad Sci U S A 2008, 105(16):6156-6161.

8. Toepel J, Welsh E, Summerfield TC, Pakrasi HB, Sherman LA: Differential transcriptional analysis of the cyanobacterium Cyanothece sp. strain ATCC 51142 during light-dark and continuous-light growth. J Bacteriol 2008, 190(11):3904-3913.

9. Min H, Sherman LA: Hydrogen production by the unicellular, diazotrophic cyanobacterium Cyanothece sp. strain ATCC 51142 under conditions of continuous light. App/ Environ Microbiol 2010, 76(13):4293-4301.

10. Aryal UK, Stockel J, Krovvidi RK, Gritsenko MA, Monroe ME, Moore RJ, Koppenaal DW, Smith RD, Pakrasi HB, Jacobs JM: Dynamic proteomic profiling of a unicellular cyanobacterium Cyanothece ATCC51142 across light-dark diurnal cycles. BMC Syst Biol 2011, 5:194.

11. Aryal UK, Stockel J, Welsh EA, Gritsenko MA, Nicora CD, Koppenaal DW, Smith RD, Pakrasi HB, Jacobs JM: Dynamic proteome analysis of Cyanothece sp. ATCC 51142 under constant light. J Proteome Res 2012, 11(2):609-619.

12. Stockel J, Jacobs JM, Elvitigala TR, Liberton M, Welsh EA, Polpitiya AD, Gritsenko MA, Nicora CD, Koppenaal DW, Smith RD, et al: Diurnal rhythms result in significant changes in the cellular protein complement in the cyanobacterium Cyanothece 51142. PLOS ONE 2011, 6(2):e16680.

13. Aryal UK, Callister SJ, McMahon BH, McCue LA, Brown J, Stockel J, Liberton M, Mishra S, Zhang X, Nicora CD, et al: Proteomic screens of five strains of oxygenic photosynthetic cyanobacteria of the genus Cyanothece. J Proteome Res 2014, 2014. doi:10.1021/pr5000889.

14. Aryal UK, Callister SJ, Mishra S, Zhang XH, Shutthanandan JI, Angel TE, Shukla AK, Monroe ME, Moore RJ, Koppenaal DW, et al: Proteome analyses of strains ATCC 51142 and PCC 7822 of the Diazotrophic Cyanobacterium Cyanothece sp. under culture conditions resulting in enhanced H-2 production. Appl Environ Microbiol 2013, 79(4):1070-1077.

15. Wegener KM, Singh AK, Jacobs JM, Elvitigala T, Welsh EA, Keren N, Gritsenko MA, Ghosh BK, Camp DG 2nd, Smith RD, et al: Global proteomics reveal an atypical strategy for carbon/nitrogen assimilation by a cyanobacterium under diverse environmental perturbations. MCP 2010, 9(12):2678-2689.

16. Allen MM: Simple conditions for growth of unicellular blue-green algae on plates. J Phycol 1968, 4(1):1-4.

17. Welkie DG, Sherman DM, Chrisler WB, Orr G, Sherman LA: Analysis of carbohydrate storage granules in the diazotrophic cyanobacterium Cyanothece sp. PCC 7822. Photosynth Res 2013, 118(1-2):25-36.

18. Kogenaru S, Qing Y, Guo Y, Wang N: RNA-seq and microarray complement each other in transcriptome profiling. BMC Genomics 2012, 13:629.

19. van Vliet $\mathrm{AH}$ : Next generation sequencing of microbial transcriptomes: challenges and opportunities. FEMS Microbiol Lett 2010, 302(1):1-7.

20. Wang Z, Gerstein M, Snyder M: RNA-Seq: a revolutionary tool for transcriptomics. Nat Rev Genet 2009, 10(1):57-63.

21. Colon-Lopez M, Sherman DM, Sherman LA: Transcriptional and translational regulation of nitrogenase in light-dark- and continuous- 
light grown cultures of the unicellular cyanobacterium Cyanothece sp. strain ATCC 51142. J Bacterio/ 1997, 179(13):4319-4327.

22. Grissa I, Vergnaud G, Pourcel C: CRISPRFinder: a web tool to identify clustered regularly interspaced short palindromic repeats. Nucleic Acids Res 2007, 35(Web Server issue):W52-W57.

23. Grissa I, Vergnaud G, Pourcel C: The CRISPRdb database and tools to display CRISPRs and to generate dictionaries of spacers and repeats. BMC Bioinformatics 2007, 8:172.

24. Waldbauer JR, Rodrigue S, Coleman ML, Chisholm SW: Transcriptome and proteome dynamics of a light-dark synchronized bacterial cell cycle. PLOS ONE 2012, 7(8):e43432.

25. Murray JW: Sequence variation at the oxygen-evolving centre of photosystem II: a new class of 'rogue' cyanobacterial D1 proteins. Photosynth Res 2012, 110(3):177-184.

26. Summerfield TC, Toepel J, Sherman LA: Low-oxygen induction of normally cryptic psbA genes in cyanobacteria. Biochemistry 2008, 47(49):12939-12941.

27. Zhang $X$, Sherman LA: Alternate copies of $D 1$ are used by cyanobacteria under different environmental conditions. Photosynth Res 2012, 114(2):133-135.

28. Barrangou $R$, Fremaux $C$, Deveau $H$, Richards M, Boyaval P, Moineau S, Romero DA, Horvath P: CRISPR provides acquired resistance against viruses in prokaryotes. Science 2007, 315(5819):1709-1712.

29. Bikard D, Jiang WY, Samai P, Hochschild A, Zhang F, Marraffini LA: Programmable repression and activation of bacterial gene expression using an engineered CRISPR-Cas system. Nucleic Acids Res 2013, 41(15):7429-7437.

30. Chylinski K, Makarova KS, Charpentier E, Koonin EV: Classification and evolution of type II CRISPR-Cas systems. Nucl Acids Res 2014, 2014 doi:10.1093/nar/gku241.

31. Garneau JE, Dupuis ME, Villion M, Romero DA, Barrangou R, Boyaval $P$, Fremaux C, Horvath P, Magadan AH, Moineau S: The CRISPR/Cas bacterial immune system cleaves bacteriophage and plasmid DNA. Nature 2010, 468(7320):67-71.

32. Makarova KS, Haft DH, Barrangou R, Brouns SJ, Charpentier E, Horvath $P$, Moineau S, Mojica FJ, Wolf YI, Yakunin AF, et al: Evolution and classification of the CRISPR-Cas systems. Nat Rev Microbiol 2011, 9(6):467-477.

33. Marraffini LA, Sontheimer EJ: CRISPR interference limits horizontal gene transfer in staphylococci by targeting DNA. Science 2008, 322(5909):1843-1845.

34. Ludwig M, Bryant DA: Transcription Profiling of the Model Cyanobacterium Synechococcus sp. Strain PCC 7002 by Next-Gen (SOLiD) Sequencing of cDNA. Front Microbiol 2011, 2:41.

35. Flaherty BL, Van Nieuwerburgh F, Head SR, Golden JW: Directional RNA deep sequencing sheds new light on the transcriptional response of Anabaena sp. strain PCC 7120 to combined-nitrogen deprivation. BMC Genomics 2011, 12:332.

36. Harke MJ, Gobler CJ: Global transcriptional responses of the toxic cyanobacterium, Microcystis aeruginosa, to nitrogen stress, phosphorus stress, and growth on organic matter. PLOS ONE 2013, 8(7):e69834.

37. Wu B, Zhang B, Feng X, Rubens JR, Huang R, Hicks LM, Pakrasi HB, Tang YJ: Alternative isoleucine synthesis pathway in cyanobacterial species. Microbiology 2010, 156(Pt 2):596-602.

38. Lan El, Liao JC: Metabolic engineering of cyanobacteria for 1-butanol production from carbon dioxide (vol 13, pg 353, 2011). Metab Eng 2012, 14(1):68-69.

39. Atsumi S, Higashide W, Liao JC: Direct photosynthetic recycling of carbon dioxide to isobutyraldehyde. Nat Biotechnol 2009, 27(12):1177-1180.

40. Bikard D, Jiang W, Samai P, Hochschild A, Zhang F, Marraffini LA: Programmable repression and activation of bacterial gene expression using an engineered CRISPR-Cas system. Nucleic Acids Res 2013, 41(15):7429-7437.

41. Qi LS, Larson MH, Gilbert LA, Doudna JA, Weissman JS, Arkin AP, Lim WA: Repurposing CRISPR as an RNA-guided platform for sequence-specific control of gene expression. Cell 2013, 152(5):1173-1183.

42. Livesay EA, Tang K, Taylor BK, Buschbach MA, Hopkins DF, LaMarche BL, Zhao R, Shen Y, Orton DJ, Moore RJ, et al: Fully automated four-column capillary LC-MS system for maximizing throughput in proteomic analyses. Anal Chem 2008, 80(1):294-302.

43. Kelly RT, Page JS, Luo Q, Moore RJ, Orton DJ, Tang K, Smith RD: Chemically etched open tubular and monolithic emitters for nanoelectrospray ionization mass spectrometry. Anal Chem 2006, 78(22):7796-7801.
44. Kim S, Gupta N, Pevzner PA: Spectral probabilities and generating functions of tandem mass spectra: a strike against decoy databases. J Proteome Res 2008, 7(8):3354-3363.

45. Elias JE, Gygi SP: Target-decoy search strategy for increased confidence in large-scale protein identifications by mass spectrometry. Nat Methods 2007, 4(3):207-214

46. Qian WJ, Liu T, Monroe ME, Strittmatter EF, Jacobs JM, Kangas LJ, Petritis K, Camp DG 2nd, Smith RD: Probability-based evaluation of peptide and protein identifications from tandem mass spectrometry and SEQUEST analysis: the human proteome. J Proteome Res 2005, 4(1):53-62.

47. Kim S, Gupta N, Pevzner PA: Spectral probabilities and generating functions of tandem mass spectra: a strike against decoy databases. J Proteome Res 2008, 7(8):3354-3363.

48. Polpitiya AD, Qian WJ, Jaitly N, Petyuk VA, Adkins JN, Camp DG 2nd, Anderson GA, Smith RD: DAnTE: a statistical tool for quantitative analysis of -omics data. Bioinformatics 2008, 24(13):1556-1558.

\section{doi:10.1186/1471-2164-15-1185}

Cite this article as: Welkie et al.: Transcriptomic and proteomic dynamics in the metabolism of a diazotrophic cyanobacterium, Cyanothece sp. PCC 7822 during a diurnal light-dark cycle. BMC Genomics 2014 15:1185.

\section{Submit your next manuscript to BioMed Central and take full advantage of:}

- Convenient online submission

- Thorough peer review

- No space constraints or color figure charges

- Immediate publication on acceptance

- Inclusion in PubMed, CAS, Scopus and Google Scholar

- Research which is freely available for redistribution
C Biomed Central 